\title{
DIVULGAÇÃO DA RESPONSABILIDADE SOCIAL DAS COOPERATIVAS AGROPECUÁRIAS BRASILEIRAS
}

\section{SOCIAL RESPONSIBILITY INFORMATION DISCLOSED BY BRAZILIAN AGRICULTURAL COOPERATIVES}

\section{DIVULGACIÓN DE LA RESPONSABILIDAD SOCIAL DE LAS COOPERATIVAS AGROPECUARIAS BRASILEÑAS}

\section{Recebimento: 12/04/2018- Aceite: 13/10/2018- Publicação: 28/10/2018 \\ Processo de Avaliação: Double Blind Review}

Jacyara Souzal

Mestre em Administração

Universidade Federal de Mato Grosso do Sul

jacy_souz@hotmail.com

\section{Rosamaria Moura-Leite}

Professora do Programa de Pós-graduação em Administração da Escola de Administração e Negócios da Universidade Federal de Mato Grosso do Sul

Universidade Federal de Mato Grosso do Sul

rosamaria.leite@ufms.br

\section{Matheus Wemerson Gomes Pereira}

Professor do Programa de Pós-graduação em Administração da Escola de Administração e Negócios da Universidade Federal de Mato Grosso do Sul

Universidade Federal de Mato Grosso do Sul

matheuswgp@yahoo.com.br

\section{RESUMO}

A divulgação do compromisso social por meio da internet vem sendo amplamente desenvolvida pelas organizações com o intuito de beneficiar tanto seu público interno, quanto externo. Em relação à cooperativas a comunicação com a comunidade tem maior importância tendo em vista relação da responsabilidade social com os princípios cooperativistas. Dessa forma o objetivo do estudo é analisar o nível de informação de responsabilidade social divulgadas pelas cooperativas agropecuárias brasileiras em seus websites institucionais. Para mensurar esse nível de informação adotou-se o instrumento desenvolvido por Muñoz e Valencia (2014), e para a análise de dados além de estatística descritivas, fez-se uso de análise fatorial e em seguida a análise de cluster. Foram analisados os websites institucionais de 31 cooperativas agropecuárias listadas no ranking das Maiores e Melhores Empresas, elaborado pela Revista Exame (2015).

\footnotetext{
${ }^{1}$ Autor para correspondência: Universidade Federal do Mato Grosso do Sul - UFMS. Av. Costa e Silva, s/nº Bairro Universitário, Campo Grande - MS. 79070-900, Brasil.
} 
Averiguou-se grande defasagem no nível de informações divulgada pelas cooperativas, já que prevalecem a divulgação de variáveis relacionadas à identificação de negócios e reconhecimentos, e não é abordada na maioria das cooperativas estudadas a forma como tem sido articulada sua política de responsabilidade social.

Palavras-Chave: Responsabilidade Social. Transparência. Internet. Cooperativas agropecuárias.

\begin{abstract}
The dissemination of social commitment through the internet has been widely developed by organizations with the aim of benefiting both their internal and external audiences. In relation to cooperatives, communication with the community is more important in view of the relationship of social responsibility with cooperative principles. Thus, the objective of the study is to analyze the level of social responsibility information disclosed by Brazilian agricultural cooperatives in their institutional websites. In order to measure this level of information, the instrument developed by Muñoz and Valencia (2014) was adopted, and for the analysis of data besides descriptive statistics, factorial analysis was used, followed by cluster analysis. Institutional websites of 31 agricultural cooperatives listed in the ranking of Largest and Best Companies, prepared by Exame Magazine (2015), were analyzed. There was a great discrepancy in the level of information disclosed by cooperatives, since the disclosure of variables related to business identification and recognition prevails, and the way in which their social responsibility policy has been articulated is not addressed in most of the cooperatives studied.
\end{abstract}

Keywords: Social Responsibility. Transparency. Internet. Agricultural cooperatives

\title{
RESUMEN
}

La divulgación del compromiso social a través de Internet viene siendo ampliamente desarrollada por las organizaciones con el objetivo de beneficiar tanto su público interno, como externo. En relación a las cooperativas, la comunicación con la comunidad tiene mayor importancia teniendo en cuenta la responsabilidad social con los principios cooperativistas. De esta forma el objetivo del estudio es analizar el nivel de información de responsabilidad social divulgadas por las cooperativas agropecuarias brasileñas en sus websites institucionales. Para medir ese nivel de información se adoptó el instrumento desarrollado por Muñoz y Valencia (2014), y para el análisis de datos además de estadística descriptiva, se hizo uso de análisis factorial y luego el análisis de cluster. Se analizaron los websites institucionales de 31 cooperativas agropecuarias listadas en el ranking de las Mayores y Mejores Empresas, elaborado por la Revista Exame (2015). Se verificó un gran desfase en el nivel de información divulgado por las cooperativas, ya que prevalecen la divulgación de variables relacionadas a la identificación de negocios y reconocimientos, y no se aborda en la mayoría de las cooperativas estudiadas la forma en que se ha articulado su política de responsabilidad social.

Palabras clave: Responsabilidad Social. Transparencia. Internet. Cooperativas agropecuarias 


\section{INTRODUÇÃO}

A cooperativa é uma associação autônoma de pessoas que se unem voluntariamente, para satisfazer aspirações e necessidades econômicas, sociais e culturais comuns, por meio de uma empresa de propriedade coletiva e democraticamente gerida. Essas pessoas baseiam-se em valores de ajuda mútua e responsabilidade, democracia, igualdade, equidade e solidariedade (OCB, 2014). O cooperativismo na tradição dos seus fundadores tem como valores a crença em princípios éticos de honestidade, abertura, responsabilidade social e preocupação com o próximo (MACPHERSON, 2003).

No Brasil, as cooperativas atuam em 13 setores da economia; no entanto, tendo em vista a importância e representatividade econômica e social na realidade brasileira, esse estudo aborda as cooperativas agropecuárias. Em maio de 2017, estavam cadastradas na OCB 1.555 cooperativas agropecuárias, somando 1.016.606 cooperados e gerando cerca de 188.777 empregos diretos (OCB, 2017).

De acordo com o último censo agropecuário realizado pelo instituto Brasileiro de Geografia e Estatística em 2006, 48\% da produção agrícola no Brasil passa de alguma forma por uma cooperativa. Mesmo em um cenário de crise na economia brasileira, as grandes cooperativas agrícolas do país conseguiram elevar suas receitas em até 15\% em 2016, e as 70 maiores do ramo agropecuário faturaram cerca de $\mathrm{R} \$ 123$ bilhões no mesmo ano, segundo estimativa da Organização das Cooperativas Brasileiras (OCB, 2017).

Baseado em diversos estudos, Pavie (2008) define a responsabilidade social como um conjunto de ações voluntárias que a empresa deve promover que visem a melhoria das condições sociais e ambientais, isso é as respostas da empresa para suas questões econômicas, técnicas e legais devem gerar benefícios sociais e ambientais, além de ganhos financeiros.

Freeman e Mc Vea (2001) ressaltam que a atuação do gestor de empresa deve ter múltiplos objetivos, isso é atender os interesses das diversas partes interessadas da organização: acionistas, empregados, clientes, fornecedores e sociedade. As partes interessadas ou stakeholders em inglês são pessoas, grupos ou entidades, que tenham relações ou interesses diretos ou indiretos com ou na empresa. As definições menos abrangentes, por sua vez, os consideram como atores sem os quais a empresa inviabilizar-se-ia, como empregados, gerentes, fornecedores, proprietários, acionistas e clientes, todos interessados e com expectativas em relação à organização (DONALDSON; PRESTON, 1995). 
Segundo, Sparemberger, Zamberlani e Savoldi (2011), é importante ressaltar que o conceito de responsabilidade social nas cooperativas está fundamentado nos valores que moldaram os primeiros movimentos cooperativistas. As cooperativas devem utilizar todas as oportunidades para conscientizar os funcionários e cooperados que o suporte as demais partes interessadas é importante não só para o seu negócio, mas também para fortalecer o desenvolvimento local integrado e sustentável (SPAREMBERGER; ZAMBERLAN; SAVOLDI, 2011).

Segundo Morsing e Schultz (2006), mensagens sobre a ética corporativa e as iniciativas sociais empresariais têm o poder de evocar fortes reações positivas entre os stakeholders. Com isso, as empresas estão cada vez mais preocupadas em comunicar aos diversos públicos que atua de forma ética e responsável.

Neste sentido, as empresas podem comunicar suas ações de RSE utilizando novas tecnologias e fazendo com que, cada vez mais pessoas tenham acesso a tais informações. Assim a internet mostra-se como uma ferramenta eficaz para disseminação das informações, sendo um canal de comunicação entre a empresa, seus clientes, fornecedores, investidores, governo e sociedade (AGONESE; SANCHES; BEZERRA, 2014). O uso da análise de websites institucionais das organizações vêm sendo adotado em estudos qualitativos e quantitativos sobre a comunicação de responsabilidade social (BARBALHO; MEDEIROS, 2014; RAHMAN; DEBRECENY, 2014)

O trabalho de Muñoz e Valencia (2014), apresenta um instrumento para análise da divulgação da responsabilidade social aplicadas em cooperativas agropecuárias espanholas. Este trabalho propõe aplicar o instrumento da pesquisa espanhola em cooperativas brasileiras, com o objetivo de analisar o nível de informação de responsabilidade social divulgadas pelas cooperativas agropecuárias brasileiras em seus websites institucionais. Trata-se de um tema relevante dado à importância econômica das cooperativas agropecuárias e da necessidade de se verificar de que forma essas organizações utilizam seus websites institucionais para se comunicarem com seus stakeholders.

Assim, o trabalho é composto de um referencial teórico sobre a temática, da exposição dos procedimentos metodológicos da pesquisa utilizada, da apresentação dos resultados, organizada em: análise descritiva, análise fatorial, análise de cluster. Em seguida apresenta-se a discussão dos resultados obtidos, demonstrando as diferenças e semelhanças encontradas entre as cooperativas analisadas, e a forma como foram agrupadas de acordo como os níveis de 
informações divulgadas na internet; e, por fim, são apresentadas as considerações finais da pesquisa.

\section{FUNDAMENTAÇÃO TEORICA}

\subsection{A RESPONSABILIDADE SOCIAL NAS COOPERATIVAS}

As cooperativas, no Brasil são definidas como organizações de fins econômicos, mas que não têm como finalidade o lucro, são constituídas por associados que, simultaneamente, são clientes, beneficiários, gestores da organização e investidores, onde as decisões são democráticas e cada cooperado possui um único voto e no caso de existir sobra, independentemente de seu tamanho, poderá ser distribuída em proporção à atividade de cada sócio para com a cooperativa. (CARVALHO; BIALOSKORSKI NETO, 2008)

Os empreendimentos cooperativos devem respeitar os princípios que norteiam o funcionamento das cooperativas no Brasil e no mundo (OCB, 2011). São elencados sete princípios, dos quais três (educação, formação e informação; cooperação com a comunidade; e interesse na comunidade) demonstram a preocupação do movimento cooperativo para atividades sociais e, consequentemente, para a própria responsabilidade social. Apesar de toda a orientação doutrinária sobre a responsabilidade social, não existe uma definição fundamental do termo como aplicado às cooperativas e não cooperativas. (BORTOLETO; COSTA, 2012)

O compartilhamento doutrinário, de acordo com Zylbersztajn (2002) embora não seja homogêneo e universal, criou as bases para uma linguagem comum, permitindo que se faça referência a um movimento cooperativista internacional, devidamente estruturado e regido, institucionalmente, pela Aliança Cooperativista Internacional.

Seus princípios empresariais afirmam que a gestão da organização deve ser eminentemente social, baseado na transparência, democracia, cooperação e gestão ambiental, entre outros. Dessa forma, a responsabilidade social está implícita na gestão destas entidades e uma das exigências da mesma, a transparência, deve ser desenvolvida através da emissão de relatórios de sustentabilidade (HORRACH; SOCIAS, 2011).

A responsabilidade social é considerada uma abordagem moderna aos problemas econômicos e sociais que surgiram com a globalização e oferece as cooperativas uma nova maneira de melhorar sua responsabilidade em relação aos seus membros. Pode-se constatar que as ações de responsabilidade social se tornam concretas, observando as mudanças benéficas geradas na comunidade ou entre os profissionais que fazem parte de uma cooperativa 
(CACCIA; ZARELLI; GIMENES, 2008). Segundo Irion (1997), a cooperativa, ao assumir uma preocupação com a sociedade, na integração com a comunidade, desempenha objetivamente sua função social, atuando diretamente no bem-estar social da comunidade, comprometendo-se com o desenvolvimento de projetos que beneficiem a comunidade local ou regional.

A revisão dos trabalhos, realizada por Bortoleto e Costa (2012), que investigaram as ações sociais das cooperativas, dentre eles as pesquisas de Mugarra (2001), Tous e Ciruela (2005), Ambrogi (2007) e Puentes e Mozas(2010), confirmam a importância dada aos princípios do cooperativismo e dos stakeholders na função objetivo das cooperativas e na concepção assumida sobre responsabilidade social.

\subsection{TRANSPARÊNCIA E DIVULGAÇÃO}

Governança corporativa, responsabilidade, transparência, comunicação e avaliação são questões que estão intimamente relacionadas, na medida em que a transparência fornece uma estrutura para melhorar a informação disponível para os stakeholders e para fornecer à empresa maior credibilidade social (MUÑOZ; TIRADO; LOZANO, 2010).

De acordo com Horrach e Socias (2011), a obrigação das organizações para desenvolver informação econômica e social é fixada em suas disposições regulamentares, em maior ou menor grau, mas depende da forma jurídica adotada. Neste sentido, a divulgação pública da informação produzida é, o primeiro passo para assumir o critério de transparência.

A transparência, como argumenta Morales e Ariza (2010) é um exercício de responsabilidade, mas ao mesmo tempo é um meio para os compromissos assumidos pela empresa se tornarem conhecidos e para que sejam aprovados por seus stakeholders. No caso das cooperativas, pode até ser instrumentalizado como um mecanismo de controle externo para justificar os níveis de apoio econômico, financeiro e fiscal alcançado a nível nacional e regional.

Através da transparência as empresas podem alcançar legitimidade, mas também pode se considerar um propósito das próprias organizações, porque gera confiança e afeta sua reputação, além de aumentar a fidelidade dos parceiros (MORALES; ARIZA,2010). As principais motivações para a divulgação de informações sobre a RSE estão relacionadas com a prestação de contas aos stakeholders e a legitimidade que contribui para a atividade da cooperativa. No entanto alguns autores ressaltam o risco reputacional, o que pode gerar uma interpretação negativa da informação (HORRACH; SOCIAS, 2011). 
As empresas serão cada vez mais pressionadas a olharem intensamente os impactos das suas operações, dentro e fora das paredes institucionais, e cuidadosamente verificarem os impactos de suas políticas e ações nos seus empregados, clientes, comunidades e na sociedade como um todo (OLIVEIRA; MOURA-LEITE, 2014). Teorias organizacionais, tais como a teoria dos stakeholders (FREEMAN, 1984), foram aplicadas na academia de contabilidade para determinar as variáveis que influenciam a divulgação (HORRACH; SOCIAS, 2011).

Segundo Holtz (2005), muitos indivíduos são consumidores de informações. Porém, antes da Internet, o acesso a essas informações era limitado a um pequeno número de pessoas. A Internet mudou esse panorama e hoje é um excelente canal de comunicação, muito utilizado para vender produtos, divulgar informações e conectar pessoas, entre outras funcionalidades. Assim, torna-se importante o uso da Internet para comunicar as informações relacionadas à responsabilidade social, já que a consciência dos consumidores e o ambiente competitivo exercem certa pressão em prol das questões sociais (SCHULTZ, CASTELLÓ, MORSING, 2013).

Transparência e prestação de contas são práticas essenciais da boa governança, o que significa que estes não só afetam diretamente o funcionamento da empresa, mas todos aqueles que contribuem para o fortalecimento do capital institucional da organização, sendo assim fundamental para a sua sustentabilidade (KANG; HUSTVEDT, 2014). No caso das cooperativas, a transparência pode até ser instrumentalizada como um mecanismo de controle externo para justificar os níveis de apoio econômico, financeiro e fiscal alcançado a nível nacional e regional (MORALES; ARIZA, 2010).

\section{PROCEDIMENTOS METODOLÓGICOS}

Trata-se de uma pesquisa que busca analisar o nível de informação de responsabilidade social divulgadas pelas cooperativas agropecuárias brasileiras em seus websites institucionais Foi adotado um instrumento de coleta de dados estruturado elaborado por Muñoz e Valencia (2014), que é composto por 32 variáveis dividas em 5 categorias. A processo de escolha das variáveis, segundo Muñoz e Valencia (2014), se deu através do Guia para a Elaboração de Relatórios de Sustentabilidade da organização internacional Global Reporting Initiative (GRI), pois atualmente é a iniciativa com maior reconhecimento internacional na área de padronização de informações de conteúdo relativas à responsabilidade social. 
Os referidos autores limitaram-se à primeira parte do documento, que inclui os aspectos gerais da empresa, sua estratégia, relações com as partes interessadas, o governo e a divulgações de parâmetros, entre outros. Todas estas questões foram agrupadas em cinco categorias, coincidindo com as primeiras quatro seções do documento GRI (2011), dividindo a última seção, por sua vez, em dois (MUÑOZ; VALENCIA, 2014). As categorias analisadas no estudo são:

Categoria 1 - Estratégia e Análise: a primeira categoria aborda a estratégia da empresa através da demonstração pessoal do presidente da organização, nomeadamente em termos de sustentabilidade e, se assim for, sobre as questões prioritárias incluídas no relatório de sustentabilidade.

Categoria 2 - Perfil da Organização: para a segunda categoria do questionário, refere-se à entidade real, examina se as empresas relatam em seu site informações tais como produtos, participação em outras empresas e estrutura corporativa, localização da sede, países produtores onde atua, forma jurídica, a sua dimensão mercados medida ou através de diferentes magnitudes, assim como a história da entidade ou premiações recebidas em termos de sustentabilidade.

Categoria 3 - Parâmetros da informação: a terceira categoria diz respeito aos parâmetros da informação transmitida sobre a sustentabilidade, a existência de um ponto de contato, o alcance e a cobertura da informação, bem como a existência de limitações no conteúdo apresentado e à existência de processos de garantia externos. No caso de não se referir a esses aspectos, pergunta se menciona como essas questões serão resolvidas no futuro.

Categoria 4 - Governança Corporativa: é a quarta categoria, e questiona o que é informado sobre as responsabilidades do conselho e do presidente, em especial, se estes aspectos estão associados a retribuição. Internamente pergunta se são relatados os mecanismos estabelecidos para evitar conflitos de interesse. Globalmente, questiona a definição da missão, declaração de princípios ou valores da organização, ou a existência de códigos de conduta e o estágio de implementação das ações. Além disso, examina se o conselho exerce funções de supervisão a questões específicas que afetam a sustentabilidade e, inversamente, se há mecanismos que controlam o conselho de administração a este respeito.

Categoria 5 - Grupos de Interesse: a quinta categoria do questionário incide sobre os stakeholders. Primeiro analisa se a cooperativa trabalha na identificação e seleção dos stakeholders, se existem canais para identificar suas demandas e questões materiais de especial 
importância e se existem mecanismos de feedback que garantam a aprendizagem. O grau de compromisso com os grupos analisados, se existem procedimentos para o tratamento das reclamações, se a gestão é avaliada periodicamente, se há comunicação dos resultados dessa avaliação, ou se os processos concretos para coordenar iniciativas satisfazem as suas expectativas.

Para cada item, foi calculada a média ponderada numérica, obtida através de quatro tipos de pontuações para cada uma das variáveis, em função da quantidade e qualidade das informações disponíveis nos sites institucionais de cada uma das cooperativas estudadas (Muñoz e Valencia, 2014). Os valores serão atribuídos da seguinte forma:

a) 0 , em casos em que a informação não é relatada ou apresentada, demonstrando falta de transparência sobre o assunto.

b) 1, em casos em que a informação é encontrada no site, demonstrando um pouco a preocupação sobre o tema.

c) 2, quando a informação disponível for genérica, onde a empresa atribui certa importância ao tema, mas não são relatados detalhes, por falta de capacidade ou recursos.

d) 3, o valor será atribuído quando a informação divulgada for completa.

Segundo Vieira e Dalmoro (2008) a escolha do número de itens na escala impacta também nos testes estatísticos realizados nas análises dos dados. O uso de uma escala com muitos pontos pode não resultar uma base de dados válida para a realização de inferências estatísticas, uma vez que o tamanho da amostra pode resultar em uma dispersão entre os respondentes e curvas fora dos padrões normais, limitando o uso de alguns testes estatísticos.

Após a coleta e tipificação das informações, foi realizada análise de frequência e médias de cada uma das variáveis e categorias.

Um processo alternativo de mensuração é identificar variáveis que apresentam a mesma estrutura subjacente. Tecnicamente, isso pode ser implementado através da análise fatorial. A principal função das diferentes técnicas de análise fatorial é reduzir uma grande quantidade de variáveis observadas a um número reduzido de fatores. Os fatores representam as dimensões latentes (construtos) que resumem ou explicam o conjunto de variáveis observadas (HAIR et al, 2006). Ao resumir dados, a análise fatorial obtém dimensões latentes que descrevem os dados em um número menor de conceitos do que as variáveis individuais originais (HAIR et al, 2006). 
Após a análise fatorial adotou-se a análise de agrupamentos, também conhecida como análise de Cluster ou de conglomerados. Trata-se de uma técnica estatística multivariada que tenta sintetizar ou simplificar a estrutura de variabilidade dos dados. O objetivo dessa técnica é organizar elementos (observações ou variáveis), em grupos, com base nas informações quanto à sua proximidade (MINGOTI, 2005).

Nesse estudo, optou-se por utilizar o método de ligação de Ward em que a distância entre dois agrupamento é a soma dos desvios quadrados dos pontos aos centroides. A meta do método de ligação de Ward é minimizar a soma dos quadrados dentro dos agrupamentos, conforme destacado por Hair et al (2006). Todas as análises foram desenvolvidas através do software Stata13.

Para o estudo foram selecionadas as 33 maiores cooperativas agropecuárias brasileiras, conforme publicação da Revista Exame: Melhores e Maiores, de julho de 2015, que relaciona as mil maiores empresas do Brasil. No entanto uma das cooperativas, a CHS do Brasil, foi excluída, pois, apesar de atuar no Brasil, trata-se de uma empresa norte americana e a CCAB Agro foi definida como um consórcio de cooperativas, desta forma não sendo de interesse neste estudo. Assim, a amostra foi composta por 31 cooperativas que tiveram os sites analisados.

Os critérios utilizados pela Revista Exame: Melhores e Maiores para avaliar as organizações são os seguintes: crescimento das vendas, liderança de mercado, liquidez corrente, liquidez geral, rentabilidade e riqueza por empregado, onde é atribuída uma pontuação a cada um dos critérios. Além disso, a organização recebe pontos por mostrar transparência na divulgação de demonstrações contábeis, como também receberá pontos extras caso figure em alguma outra publicação relacionada, da mesma editora, como: o Guia Exame de sustentabilidade, o Guia Você S/A - As melhores empresas para você trabalhar.

A escolha das cooperativas da amostra com base no ranking desenvolvido pela publicação, contribui com o objetivo deste trabalho, que consiste analisar o nível de informação de responsabilidade social divulgadas pelas cooperativas agropecuárias brasileiras em seus websites institucionais. As organizações maiores têm mais recursos e, portanto, são mais propensas a ter o seu próprio site e pessoal necessário para manter conteúdo mais amplo e atualizado. A coleta de dados ocorreu entre os meses de novembro e dezembro de 2015.

\section{APRESENTAÇÃO DOS RESULTADOS}


A seguir são apresentados os resultados obtidos através de analises estatísticas. Primeiramente, apresenta-se análise descritiva onde observam-se as frequências e médias obtidas para cada variável contemplada e categorias, prosseguindo para a Análise Fatorial com a extração dos fatores, e através dos escores dessa última foi realizada a Análise de Cluster, onde as cooperativas foram agrupadas de acordo com as semelhanças encontradas nos níveis de informações divulgados.

\subsection{ANÁLISE DESCRITIVA}

Os resultados obtidos sobre as frequências para cada uma das 32 variáveis do estudo estão resumidos na Tabela 1, em conjunto com a média e desvio padrão de cada variável.

Tabela 1:As frequências das variáveis

\begin{tabular}{|c|c|c|c|c|}
\hline & CATEGORIA 1. ESTRATÉGIA E ANALISE & Freq & Média & $\begin{array}{l}\text { Desv. } \\
\text { Padrão }\end{array}$ \\
\hline 1 & $\begin{array}{l}\text { Há uma declaração do responsável máximo sobre a relevância da sustentabilidade para a } \\
\text { organização }\end{array}$ & $15 \%$ & 0,45 & 0,96 \\
\hline 2 & $\begin{array}{l}\text { As prioridades estratégicas e questões -chave sobre a sustentabilidade a médio / curto } \\
\text { prazo e sua relação com longa }\end{array}$ & $17 \%$ & 0,52 & 0,72 \\
\hline 3 & Eventos principais, conquistas e fracassos & $31 \%$ & 0,94 & 0,92 \\
\hline 4 & Perspectivas nível de conformidade em relação aos objetivos & $12 \%$ & 0,35 & 0,75 \\
\hline 5 & $\begin{array}{l}\text { Descrição dos principais impactos, riscos e oportunidades para a empresa, e seu impacto } \\
\text { sobre as partes interessadas }\end{array}$ & $14 \%$ & 0,42 & 0,72 \\
\hline \multirow[t]{2}{*}{6} & $\begin{array}{l}\text { O impacto sobre as perspectivas de longo prazo e ao desempenho financeiro da } \\
\text { organização }\end{array}$ & $5 \%$ & 0,16 & 0,45 \\
\hline & CATEGORIA 2. PERFIL DA ORGANIZAÇÃO & & & \\
\hline 7 & $\begin{array}{l}\text { Seus produtos são comercialmente identificados no mercado por meio de sua própria } \\
\text { marca ou comerciante }\end{array}$ & $60 \%$ & 1,81 & 1,24 \\
\hline 8 & Áreas de responsabilidade e de participações são identificados & $30 \%$ & 0,90 & 0,74 \\
\hline 9 & A localização da sede da entidade e sua produção é identificado & $59 \%$ & 1,77 & 0,95 \\
\hline 10 & $\begin{array}{l}\text { Os países em que operam estão identificados e citados os aspectos mais relevantes da } \\
\text { sustentabilidade }\end{array}$ & $11 \%$ & 0,32 & 0,74 \\
\hline 11 & A forma jurídica da entidade indicada & $40 \%$ & 1,19 & 0,74 \\
\hline 12 & Mercados atendidos (por países, setores e tipos de clientes) & $10 \%$ & 0,29 & 0,69 \\
\hline 13 & $\begin{array}{l}\text { Dimensão (empregados, as vendas, a dívida, a quantidade de produto) e outros (ativos } \\
\text { proprietários, repartição por país / região) }\end{array}$ & $45 \%$ & 1,35 & 1,19 \\
\hline 14 & $\begin{array}{l}\text { Os relatórios sobre mudanças significativas ocorreram ao longo da história da } \\
\text { organização }\end{array}$ & $24 \%$ & 0,71 & 0,82 \\
\hline \multirow[t]{2}{*}{15} & Relatórios dos prêmios ou distinções obtidas & $24 \%$ & 0,71 & 1,21 \\
\hline & CATEGORIA 3. PARÂMETROS DE INFORMAÇÃO & & & \\
\hline 16 & A data do último relatório publicado CSR ou Sustentabilidade é identificado (se houver) & $23 \%$ & 0,68 & 1,10 \\
\hline 17 & Há ponto geral de contato para questões relacionadas com as informações fornecidas & $97 \%$ & 2,90 & 0,53 \\
\hline 18 & $\begin{array}{l}\text { Define o conteúdo econômico, social e ambiental, prioriza aspectos e identifica } \\
\text { claramente as partes interessadas. }\end{array}$ & $38 \%$ & 1,13 & 0,99 \\
\hline
\end{tabular}




\begin{tabular}{|c|c|c|c|c|}
\hline 19 & $\begin{array}{l}\text { Se não cobre todos os impactos econômicos, sociais e ambientais, indica de que forma } \\
\text { serão alcançados. }\end{array}$ & $10 \%$ & 0,29 & 0,82 \\
\hline & CATEGORIA 4. GOVERNANÇA CORPORATIVA & & & \\
\hline 20 & $\begin{array}{l}\text { Composição do Conselho e as responsabilidades atribuídas em questões econômicas, } \\
\text { sociais e ambientais }\end{array}$ & $29 \%$ & 0,87 & 0,34 \\
\hline 21 & Ele informa se o presidente detém cargo executivo e suas funções & $9 \%$ & 0,26 & 0,51 \\
\hline 22 & $\begin{array}{l}\text { Existe uma relação entre remuneração e desempenho econômico, social e ambiental dos } \\
\text { conselheiros, administradores e executivos }\end{array}$ & $1 \%$ & 0,03 & 0,18 \\
\hline 23 & Existem procedimentos para evitar conflitos de interesses no órgão superior & $0 \%$ & 0,00 & 0,00 \\
\hline 24 & $\begin{array}{l}\text { Missão e valores, códigos de conduta, princípios relevantes em relação à } \\
\text { sustentabilidade e estágio de sua implementação. }\end{array}$ & $32 \%$ & 0,97 & 0,65 \\
\hline 25 & $\begin{array}{l}\text { Os procedimentos do Conselho para monitorar a sustentabilidade da organização, riscos } \\
\text { e oportunidades, e de conformidade com as normas internacionais }\end{array}$ & $1 \%$ & 0,03 & 0,18 \\
\hline \multirow[t]{2}{*}{26} & $\begin{array}{l}\text { Processos para a avaliação do Conselho, em especial no que diz respeito ao desempenho } \\
\text { econômico, ambiental e social. }\end{array}$ & $0 \%$ & 0,00 & 0,00 \\
\hline & CATEGORIA 5. STAKEHOLDERS & & & \\
\hline 27 & $\begin{array}{l}\text { Estabelece a base para a identificação e seleção de stakeholders com os quais se } \\
\text { engajar. }\end{array}$ & $10 \%$ & 0,29 & 0,46 \\
\hline 28 & $\begin{array}{l}\text { Aspectos de interesse que surgiram através da participação das partes interessadas, e } \\
\text { como a organização tem adotado. }\end{array}$ & $0 \%$ & 0,00 & 0,00 \\
\hline 29 & $\begin{array}{l}\text { NGO1 Compromisso com a sociedade por meio de processos para o envolvimento das } \\
\text { partes interessadas afetadas no desenho, implementação, monitoramento e avaliação de } \\
\text { políticas e programas }\end{array}$ & $19 \%$ & 0,58 & 0,80 \\
\hline 30 & $\begin{array}{l}\text { NGO2 Comentários, reclamações e mecanismos de feedback em matéria de não } \\
\text { conformidade com as políticas e programas destinados stakeholders }\end{array}$ & $8 \%$ & 0,23 & 0,42 \\
\hline 31 & $\begin{array}{l}\text { NGO3 Há sistemas de medição (incluindo a sua eficácia), avaliação e aprendizagem e } \\
\text { comunicação de resultados para as partes interessadas }\end{array}$ & $2 \%$ & 0,06 & 0,36 \\
\hline 32 & $\begin{array}{l}\text { NGO6 Estabelecimento de processos para a coordenação de esforços para satisfazer as } \\
\text { expectativas dos diferentes intervenientes }\end{array}$ & $0 \%$ & 0,00 & 0,00 \\
\hline
\end{tabular}

Fonte: Dados da pesquisa. Elaborado pelos autores

Os resultados na Tabela 1 mostram níveis de difusão escassos acerca das informações sobre responsabilidade social, observa-se que apenas três variáveis se destacam com porcentagens superiores a $50 \%$ da frequência. A variável 17, relacionados com a existência de um ponto de contato na web, tem uma alta taxa de presença, onde $97 \%$ dos sites pesquisados apresentam este item. No entanto, não é possível verificar a qualidade das respostas, o tempo de demora ou a solução de questões dadas às partes interessadas por este meio. Os produtos comercializados são identificados e descritos na web pela cooperativa através de suas marcas, em $60 \%$ dos casos. A variável 9 , revela que $59 \%$ das cooperativas informam a localização da sede e principais centros de produção.

Tendo como base os números apresentados pode-se afirmar que há assuntos que não são objeto de comunicação na internet, referem-se principalmente a Governança Corporativa, como: os procedimentos para avaliar o desempenho social e econômico; procedimentos para evitar conflitos de interesses, não obtiveram pontuação na análise. Caso semelhante ocorre em 
relação a Categoria 5, "Stakeholders", a variável sobre os aspectos de interesse que surgiram através da participação dos stakeholders, bem como o estabelecimento de processos para coordenação para satisfação das expectativas dos diferentes stakeholders, também não tiveram pontuação

Tabela 2:Pontuação geral de acordo com a categoria.

\begin{tabular}{lcc}
\hline & Pontuação Geral & Percentual \\
\hline CATEGORIA 1. ESTRATÉGIA E ANALISE & 88 & $3 \%$ \\
CATEGORIA 2. PERFIL DA ORGANIZAÇÃO & 281 & $9 \%$ \\
CATEGORIA 3. PARÂMETROS DE INFORMAÇÃO & 155 & $5 \%$ \\
CATEGORIA 4. GOVERNANÇA CORPORATIVA & 67 & $2 \%$ \\
CATEGORIA 5. STAKEHOLDERS & 36 & $1 \%$ \\
\hline
\end{tabular}

Fonte: Dados da pesquisa. Elaborado pelos autores

Observa-se a baixa pontuação das cooperativas brasileiras nas 5 categorias estudadas. A categoria 2. "Perfil da organização", obteve a maior pontuação chegando a 9\% no nível de informação divulgada. Nota-se que a categoria com a menor pontuação é a Categoria 5 "Stakeholders", com uma taxa de 1\%. Da mesma forma pontuações baixas são observadas nas Categorias 4 "Governança Corporativa" (2\%) e a Categorias 1 "Estratégia e Análise" (3\%). A Categoria 3 "Parâmetros de Informação" está em um nível intermediário de 5\%.

\subsection{ANÁLISE FATORIAL}

Para complementar a análise descritiva foi utilizada a técnica estatística de Análise Fatorial. Como é possível observar ainda na tabela 1, 4 das variáveis em estudo $(23,26,28$ e 32), contidas nas categorias 4 e 5 que se referem a Governança Corporativa e Stakeholders respectivamente, apresentam variância igual a 0 , ou seja, todas as cooperativas apresentam a mesma resposta (0) para essas questões. Dessa forma, os dados ao serem inseridos no software são desprezados automaticamente.

Ao aplicar a análise fatorial, utilizando o software StataSE 13.0, obteve-se a extração de 8 fatores, com base no seu autovalor (eigenvalues), definido por Hair et al. (2006) como sendo a quantidade de variância associada ao fator. Juntos esses fatores são capazes de explicar $83 \%$ da variância total do modelo, conforme demonstrado na tabela 3. 
O critério utilizado para a retenção fatorial foi o critério de Kaiser-Guttman, conhecido como eigenvalue $>1$, que propõe uma avaliação rápida e objetiva do número de fatores a ser retido (PATILET AL, 2008).

Tabela 3: Extração dos fatores

\begin{tabular}{lrrrr}
\hline Fator & Variância & Diferença & Percentual & $\begin{array}{c}\text { Perc. } \\
\text { Acumulado }\end{array}$ \\
\hline Fator 1 & 6,24 & 2,88 & $22 \%$ & $22 \%$ \\
Fator 2 & 3,36 & 0,04 & $12 \%$ & $33 \%$ \\
Fator 3 & 3,32 & 0,25 & $11 \%$ & $44 \%$ \\
Fator 4 & 3,06 & 0,39 & $11 \%$ & $55 \%$ \\
Fator 5 & 2,67 & 0,51 & $9 \%$ & $64 \%$ \\
Fator 6 & 2,16 & 0,51 & $7 \%$ & $72 \%$ \\
Fator 7 & 1,65 & 0,02 & $6 \%$ & $78 \%$ \\
Fator 8 & 1,64 & 0 & $6 \%$ & $83 \%$ \\
\hline \multicolumn{5}{l}{ Fonte: Dados da pesquisa. Elaborado pelos autores }
\end{tabular}

Uma ferramenta importante na interpretação dos fatores obtidos com a análise fatorial é a sua rotação, que pode ser ortogonal ou oblíqua. Hoffmann (1999), define como objetivo da rotação ortogonal a obtenção de uma estrutura simples, isto é, abarcar uma nova matriz de coeficientes de fatores, de maneira que os valores absolutos dos componentes de cada coluna dessa matriz se aproximem, na medida do possível, de 0 ou 1 . Esse procedimento facilita a interpretação dos fatores, pois após a rotação cada fator, deve apresentar uma correlação relativamente forte com uma ou mais variáveis e correlação relativamente fraca com as demais variáveis. Entre os métodos de rotação ortogonal destaca-se o VARIMAX, mais comumente utilizado, minimiza o número de variáveis, com altas cargas sobre o fator, reforçando a compreensão dos fatores (HAIR, et al 2006). 
Tabela 4: Relação entre Fatores e Variáveis.

\begin{tabular}{|c|c|c|c|c|c|c|c|c|c|}
\hline Variáveis & Fator 1 & Fator 2 & Fator 3 & Fator 4 & Fator 5 & Fator 6 & Fator 7 & Fator 8 & Comunalidade \\
\hline V.1 & 0,62 & 0,35 & 0,05 & 0,15 & $-0,33$ & $-0,01$ & 0,04 & $-0,07$ & 0,65 \\
\hline V.2 & 0,51 & 0,33 & $-0,16$ & 0,28 & 0,18 & 0,25 & 0,56 & 0,10 & 0,89 \\
\hline V.3 & 0,54 & 0,21 & 0,33 & $-0,08$ & 0,52 & 0,38 & 0,01 & 0,11 & 0,87 \\
\hline V.4 & 0,91 & 0,09 & $-0,02$ & 0,16 & 0,00 & 0,12 & 0,05 & 0,20 & 0,93 \\
\hline V.5 & $\mathbf{0 , 8 1}$ & 0,31 & 0,13 & 0,17 & 0,19 & $-0,05$ & 0,02 & 0,22 & 0,89 \\
\hline V.6 & 0,95 & 0,10 & 0,02 & 0,07 & 0,14 & $-0,04$ & 0,01 & $-0,04$ & 0,94 \\
\hline V.7 & 0,08 & 0,62 & 0,39 & $-0,12$ & 0,24 & 0,15 & $-0,13$ & $-0,12$ & 0,67 \\
\hline V.8 & 0,16 & 0,20 & 0,41 & 0,70 & $-0,01$ & $-0,43$ & $-0,15$ & 0,10 & 0,94 \\
\hline V.9 & 0,14 & 0,61 & 0,51 & $-0,09$ & 0,05 & 0,11 & 0,41 & 0,08 & 0,84 \\
\hline V.10 & 0,02 & $-0,03$ & $\mathbf{0 , 8 0}$ & 0,02 & $-0,03$ & 0,31 & $-0,17$ & $-0,15$ & 0,79 \\
\hline V.11 & 0,04 & 0,14 & 0,91 & 0,19 & $-0,02$ & 0,10 & 0,07 & 0,11 & 0,92 \\
\hline V.12 & 0,07 & 0,11 & 0,73 & 0,44 & 0,35 & 0,15 & 0,25 & $-0,03$ & 0,95 \\
\hline V.13 & 0,27 & 0,67 & 0,10 & 0,11 & 0,38 & 0,20 & 0,06 & 0,32 & 0,84 \\
\hline V.14 & 0,31 & 0,22 & 0,04 & 0,18 & 0,76 & $-0,05$ & 0,13 & 0,14 & 0,79 \\
\hline V.15 & 0,52 & 0,05 & 0,38 & 0,13 & 0,17 & $-0,03$ & 0,02 & 0,58 & 0,79 \\
\hline V.16 & 0,51 & 0,65 & $-0,15$ & 0,34 & $-0,09$ & 0,17 & 0,00 & $-0,03$ & 0,86 \\
\hline V.17 & 0,13 & $-0,03$ & 0,03 & 0,15 & $-0,20$ & 0,06 & $-0,79$ & 0,23 & 0,76 \\
\hline V.18 & 0,45 & 0,49 & $-0,15$ & 0,46 & 0,26 & 0,04 & $-0,09$ & 0,21 & 0,79 \\
\hline V.19 & $\mathbf{0 , 8 7}$ & 0,10 & 0,13 & 0,29 & 0,17 & 0,08 & 0,02 & $-0,08$ & 0,91 \\
\hline V.20 & 0,17 & $-0,21$ & 0,14 & 0,10 & $-0,22$ & $-0,02$ & $\mathbf{0 , 6 0}$ & 0,34 & 0,63 \\
\hline V.21 & 0,09 & $-0,13$ & 0,29 & 0,06 & $-0,29$ & 0,69 & 0,11 & 0,13 & 0,7 \\
\hline V.22 & $\mathbf{0 , 8 2}$ & 0,16 & $-0,02$ & $-0,32$ & 0,09 & $-0,17$ & 0,01 & $-0,06$ & 0,85 \\
\hline V.24 & 0,12 & $\mathbf{0 , 8 3}$ & 0,11 & 0,06 & 0,09 & $-0,26$ & $-0,04$ & 0,02 & 0,79 \\
\hline V.25 & $-0,06$ & 0,09 & $-0,11$ & $-0,03$ & 0,06 & 0,00 & $-0,03$ & $\mathbf{0 , 8 4}$ & 0,73 \\
\hline V.27 & 0,36 & 0,62 & $-0,35$ & $-0,02$ & $-0,30$ & $-0,07$ & $-0,04$ & 0,26 & 0,8 \\
\hline V.29 & 0,52 & $\mathbf{0 , 6 0}$ & 0,16 & 0,16 & 0,41 & $-0,05$ & 0,16 & $-0,12$ & 0,89 \\
\hline V.30 & $-0,09$ & 0,10 & 0,25 & 0,03 & 0,23 & 0,72 & $-0,11$ & $-0,11$ & 0,68 \\
\hline V.31 & 0,29 & $-0,02$ & 0,36 & 0,72 & 0,15 & 0,32 & 0,04 & $-0,08$ & 0,87 \\
\hline
\end{tabular}

Fonte: Dados da pesquisa. Elaborado pelos autores

Considerando o critério da significância, que sugere sejam consideradas de significância para as cargas fatoriais superiores a 0,50, foi possível destacar as variáveis significativas em cada fator. As variáveis com maiores coeficientes são mais correlacionadas com o fator (MINGOTI, 2005).

Conforme observado na tabela 4, com relação às cargas fatoriais do fator 1 , denominado "Estratégia e Objetivos", verifica-se que as variáveis V1, V3, V4, V5 e V6 apresentaram uma forte correlação com este fator. Essas variáveis, que expressam a Estratégia adotada pela 
cooperativa sobre a sustentabilidade e a descrição dos objetivos organizacionais, o prazo e a forma como os resultados serão alcançados.

O Fator 2 está associado às variáveis V7, V9, V13, V16, V24, V27 e V29, este conjunto contém elementos que dizem respeito ao perfil, missão e processos envolvendo os stakeholders da cooperativa. Devido a heterogeneidade das categorias denominou-se de Fator de "Divulgação e processos."

As variáveis V10, V11 e V12, mostraram-se relacionadas ao Fator 3 “Atividades”. As informações contidas nestas questões dizem respeito a atividade da cooperativa.

As variáveis destacadas no quarto fator fazem referência, às áreas de responsabilidade V8 e sistemas de medição, avaliação e aprendizagem e comunicação de resultados para os stakeholders V31. Ao identificar cada área de responsabilidade, pode-se perceber maior compromisso com a gestão empresarial tendo em vista desempenho e a comunicação dos resultados.

O Fator 5 denominado "História" está relacionado apenas a variável V14, referente as mudanças ocorridas na empresa, contendo as principais conquistas, fracassos e mudanças.

As questões sobre o presidente da cooperativa, cargo e suas funções, além da forma como a cooperativa administra os contatos realizados pelos stakeholders, observados as variáveis V21 e V30 diretamente relacionadas ao Fator 6 "Estrutura Administrativa".

O Fator 7, relaciona-se às variáveis V2, que diz respeito a prioridades estratégicas sobre sustentabilidade e V20 relativa a composição do conselho e suas responsabilidades. Ressaltando que a correlação da variável V17, correspondente ao ponto de contato com a empresa foi negativa $(-0,79)$ em relação a este fator, ou seja, possuem um comportamento contrário.

O Fator 8 está ligado às varáveis V15, os prêmios conquistados, e V25, procedimentos para o monitoramento da sustentabilidade na organização. A relação entre essas variáveis pode indicar a ligação entre a preocupação da cooperativa em relação a sustentabilidade e o reconhecimento da adoção de procedimentos que monitorem suas ações para atingir esses objetivos.

A variável V18, questiona a definição do conteúdo econômico, social e ambiental e se a empresa prioriza aspectos e identifica claramente seus stakeholders, apresentou uma carga fatorial baixa (inferior a 0,50), ou seja, a variável não permite interpretação. 


\subsection{ANÁLISE DE CLUSTER}

A análise de Cluster busca agrupar elementos de dados baseando-se na similaridade entre eles. Os grupos são determinados de forma a obter-se homogeneidade dentro dos grupos e heterogeneidade entre eles. Obtidos os escores fatoriais, para cada uma das 31 cooperativas, em relação a cada fator, executou-se a análise de Cluster pelo método de ligação de Ward, o que constituiu os seguintes grupos homogêneos: Grupo 1 "Desempenho Intermediário" - com 13 cooperativas; Grupo 2 - "Desempenho Inferior" com 17 cooperativa; e, Grupo 3 "Desempenho Relevante", com 1 cooperativa.

Como já mencionado anteriormente, em contexto geral, a amostra observada se comporta de maneira semelhante, o que as individualiza são os níveis de divulgação, ou seja, as categorias com maiores pontuações são as mesmas, no entanto, algumas cooperativas apresentam informações mais completas que outras assumindo assim maior destaque.

A análise detalhada desses agrupamentos, com relação às variáveis e aos escores que constituíram a análise fatorial, apontou que o grupo formado por 13 cooperativas, foi denominado "Desempenho Intermediário", tendo em vista os escores obtidos, por sua vez maiores que os definidos no grupo 2 e em sua maioria menores aos do grupo 3. Destacando a relação determinada pela forte conexão com o Fator 2 "Divulgação e processos".

O grupo 1, demonstra que são empresas que, apesar de possuírem a preocupação com a transparência e RSE, ainda têm dificuldades em divulgar esses dados. Poderia ser a falta de uma estratégia na exposição dos dados, principalmente a não utilização do site da empresa como ferramenta de comunicação com os stakeholders.

Convém observar, conforme Muñoz et al (2010), que a governança corporativa, responsabilidade, transparência, comunicação e avaliação são questões que estão intimamente relacionadas na medida em que a transparência fornece uma estrutura para melhorar a informação disponível para as partes interessadas e para fornecer à empresa maior credibilidade social. Apesar de já haver uma estrutura para a divulgação de informação, essa plataforma deve ser aperfeiçoada.

O segundo grupo constituído por 17 cooperativas, possui os menores escores entre os fatores. Verifica-se uma certa preocupação com a comunicação, mas a qualidade dessa informação ainda é baixa. E segundo ressalta Kang e Hustvedt (2014) a transparência é um 
exercício de responsabilidade, mas ao mesmo tempo é um meio para os compromissos assumidos pela empresa serem conhecidos e aprovados por seus stakeholders.

Abaixo a Figura 1,o dendograma da análise de cluster, ilustra as relações mencionadas.

Figura 1:Dendograma obtido através do agrupamento das cooperativas de acordo com os fatores

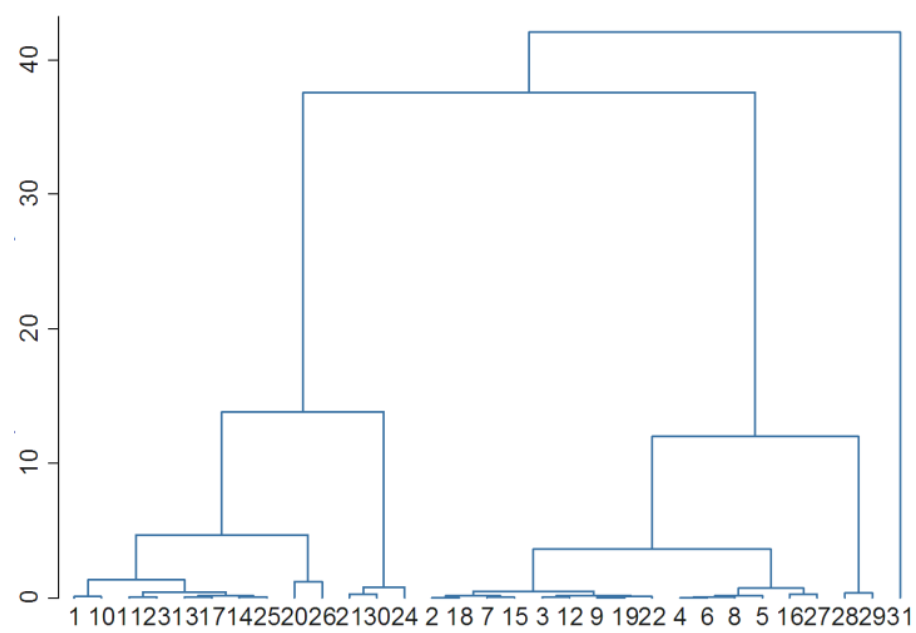

Fonte: Dados da pesquisa. Elaborado pelos autores.

Por último o Grupo 3 - "Desempenho Relevante" contendo 1 cooperativa, formado em razão de pertencerem a esta os mais altos escores fatoriais, em relação a maior parte dos fatores, com exceção dos fatores 3 "Atividades" e Fator 6 "Estrutura Administrativa”. Demonstrando que apesar de ser a organização com maior transparência em relação as suas ações, a mesma possui fragilidades.

\section{DISCUSSÃO}

A transparência da informação de responsabilidade social é um tema de pesquisa de grande interesse atual e vem sendo aplicadas em vários segmentos nas esferas pública e privada (NAVARRO; ORTIZ; ALCARAZ, 2016; SOLANO; CASADO; UREBA, 2015).

Muitas organizações e governos reconheceram as oportunidades oferecida pela web para aumentar a eficiência, transparência e competitividade (NAVARRO; ORTIZ; ALCARAZ 2014). Na pesquisa realizada observa-se que em geral, as informações nos websites institucionais das cooperativas agropecuárias aparecem de forma dispersa e fragmentada, e não 
há uniformidade no conteúdo. Observa-se que as organizações não vislumbram os websites como um meio de comunicação e diálogo com stakeholders, a fim de melhorar a transparência.

Resultado semelhante foi apresentado no estudo de Navarro, Alcaraz e Ortiz (2014) tratando das administrações públicas espanholas, onde conclui que as práticas de divulgação de informações sobre responsabilidade social são muito escassas, e não existe uma regulamentação legal que os oriente na definição e divulgação sistemática de seus compromissos. O mesmo ocorre com as cooperativas brasileiras.

Em relação à pontuação por categoria de dados, o estudo desenvolvido por Muñoz e Valencia (2014), obteve resultados semelhantes. As categorias com maiores pontuações foram as mesmas, no entanto os valores obtidos na Espanha foram muito superiores aos que constatamos no Brasil. Em relação as informações sobre o Perfil da organização e Parâmetro de informações as pontuações somam $40 \%$ e $31 \%$, respectivamente. Enquanto as cooperativas brasileiras obtiveram $9 \%$ e $5 \%$ dos pontos de divulgação nas mesmas categorias.

Apesar da defasagem em relação às cooperativas espanholas, constata-se a mesma realidade: a internet não está configurada como um meio de comunicação e diálogo com os stakeholders, a fim de melhorar a transparência, mas como uma plataforma para vender seus produtos.

No estudo desenvolvido por Kirat (2015) sobre as práticas de transparência e responsabilidade social de empresas locais do ramo de petróleo e gás do Qatar conclui que a responsabilidade social não está institucionalizada e que a divulgação das práticas realizadas não é realizada. $\mathrm{O}$ autor ressalta que o enfoque empresarial é geralmente priorizado em detrimento do social nesse setor.

Em relação às cooperativas agropecuárias, não é possível confirmar de forma conclusiva que os valores implícitos nos princípios cooperativos e os resultados positivos das suas políticas de responsabilidade social resultam de uma forma decisiva para níveis significativos de transparência. Os resultados da presente pesquisa sugerem ser necessário aumentar a conscientização dos gestores sobre as vantagens da divulgação da responsabilidade social como forma de obter vantagem competitiva. Já que de acordo com Solano, Casado e Ureba (2015) pode-se adquirir habilidades gerando vantagem competitiva que permitirá a organização melhorar sua reputação, as relações com a sociedade ou a comunidade local, aumentando a satisfação dos stakeholders, bem como aumentar a motivação de funcionários, reforçando os valores da cultura da cooperativa. 


\section{CONSIDERAÇÕES FINAIS}

Este trabalho analisa a informação de responsabilidade social divulgadas nos websites institucionais das cooperativas agropecuárias brasileiras. $\mathrm{O}$ estudo confirmou a existência de baixos níveis na divulgação de tais informações na maioria das cooperativas estudadas. Ao analisar a categoria de informações que atingiu maior pontuação revela que estes são principalmente relacionados com a identificação da organização e sua principal atividade, no entanto, são questões que têm pouca relevância em face da responsabilidade social das cooperativas. Esses são aspectos que não revelam a existência de políticas de responsabilidade social, ou os compromissos assumidos pela cooperativa com os seus stakeholders em termos de sustentabilidade.

As cooperativas agropecuárias brasileiras estão perdendo as oportunidades oferecidas pela internet como ferramenta de transparência. Ao analisar a divulgação das 31 maiores cooperativas agropecuárias brasileiras esperava-se um nível de divulgação superior já que tratase de organizações de grande porte, que dispõem de recursos para a manutenção e atualização do conteúdo de seus websites institucionais.

As questões relacionadas, principalmente, à Governança e a forma como a cooperativa se relaciona com seus stakeholders não são abordados na internet. Ressalta-se ainda a lacuna encontrada sobre os procedimentos para conflitos de interesse e para avaliar o conselho no que diz respeito ao desempenho econômico, social e ambiental, como também em relação aos aspectos que tenham surgido da interação com os stakeholders e os processos para satisfação das expectativas.

Dentre os três agrupamentos encontrados, observam-se características semelhantes, porém em níveis diferentes, um dos grupos se destacou na divulgação das informações de RSE, e os outros grupos ainda precisam melhorar a qualidade da informação publicada. No entanto é nítida a necessidade de avançar em relação as questões de governança corporativa e as relações com os stakeholders em todos os agrupamentos identificados.

Como indicação para estudos futuros, sugere-se comparar o nível de informação de responsabilidade social divulgados por outros tipos de organizações agropecuárias e cooperativas de outros setores, além da realização de estudos longitudinais que possibilitem a observação da dinâmica de divulgação dos websites estudados. 


\section{REFERÊNCIAS}

AGONESE, R.; SANCHES, J. R.; BEZERRA, F. A. Determinantes da divulgação de informações voluntárias por meio da internet. Contexto, v. 14, p. 19-31, 2014.

AMBROGI, A. A responsabilidade social das sociedades cooperativas brasileiras: uma proposta de uso da demonstração do valor adicionado - DVA. 2007. 116 f. Dissertação (Mestrado em Controladoria e Contabilidade) - Faculdade Escola de Comércio Álvares Penteado, São Paulo, 2007.

BARBALHO, F. A.; MEDEIROS, J. J. Transparência e legitimação de objetivos institucionais em empresas estatais: um estudo de caso sobre a Petrobras. Cadernos EBAPE.BR, v. 12, n. Edição Especial, p. 469-469, 2014.

BORTOLETO, F. C.; COSTA, D. R. M. The importance of Cooperatives' Corporate Social Responsibility to th eLoyalty of Members. Journal of Rural Cooperation, v. 40, p. 198-216, 2012.

CACCIA, C. Z.; ZARELLI, F. M.; GIMENES, F. M. A Prática da Responsabilidade Social das Cooperativas Agroindustriais Paranaenses COAMO E COCAMAR. Rev. Ciênc. Empres. UNIPAR, v. 9, n. 1, 2008.

CARVALHO, F. L.; BIALOSKORSKI NETO, S. Indicadores de avaliação de desempenho econômico em cooperativas agropecuárias: um estudo em cooperativas paulistas. Organizações Rurais e Agroindustriais, v. 10, n. 3, p. 420-437, 2008.

DONALDSON, T.; PRESTON, L. E. The stakeholder theory of the corporation: concepts, evidence and implications. Academy of Management Review, v.20, n.1, p.65-91, 1995.

EXAME. Melhores e maiores: as 1000 maiores empresas do Brasil, São Paulo: Editora Abril, 2015.

FREEMAN, R. E. Strategic management: a stakeholder approach. Boston: Pitman, 1984.

FREEMAN, R.E.; MC VEA, J. A stakeholder approach to Strategic Management. Darden Graduate School of Business Administration. Working Paper n. 01-02, 2001.

HAIR, JR.; BLACK, W. C.; BABIN, B. J.; ANDERSON, R. E.; TATHAM, R. L. Multivariate Data Analysis. $6^{\text {a }}$ edição. Upper Saddle River, NJ: Pearson Prentice Hall, 2006.

HOFFMANN, R. Componentes principais e análise fatorial. Piracicaba: ESALQ. 1999.

HOLTZ, S. The impactof new technologies on internal communication. Strategic Communication Management, v.10, n.1, 2005. 
HORRACH, P.; SOCIAS, A. La actitud de las empresas de economía solidaria frente a la divulgación de información sobre sostenibilidad desde el prisma de la Teoría de los Stakeholders, Revista de Contabilidad, n. 14,v. 1, p. 267-297, 2011.

KANG, J.; HUSTVEDT, G. Building trust between consumers and corporations: the role os consumer perceptions of transparency and social responsability. Journal of business ethics, v. 125, p. 253-265, 2014.

KIRAT, M. Corporate social responsibility in the oil and gas industry in Qatar perceptions and practices. Public Relations Review, v.41, p.438-446, 2015.

MACPHERSON, I. Princípios cooperativos para o século XXI. Tradução de Clarissa Ristoff. Porto Alegre: Lagoa, 2003.

MINGOTI, S. A. Análise de Dados Através de Métodos de Estatística Multivariada: uma abordagem aplicada. Belo Horizonte: Editora UFMG, 2005.

MORALES, A. C.; ARIZA, J. A. M. Sistemas de Gobierno Corporativo en las Empresas Propiedad de los Trabajadores. Revista de la Responsabilidad Social de la Empresa, n. 5, 2010.

MORSING, M.SCHULTZ, M. Corporate social responsibility communication: Stakeholder information, response and involvement strategies. Business Ethics - A European Review, n.15, v.4, p.323-338, 2006.

PUENTES P.R..; MOZAS. M. A. La responsabilidad social corporativa y su paralelismo con las sociedades cooperativas. Revesco. Revista de Estudios Cooperativos, n.103, p. 75$100,2010$.

MUGARRA, A. Responsabilidad y Balance Social hoy en día: Un Reto para las Cooperativas, Revista de Economía Pública, Social y Cooperativa. N. 39, p. 25-50, 2001.

MUÑOZ, R. M.; TIRADO, P. V.; LOZANO, M. R. Una propuesta de herramientas para la gestión de la transparencia en las entidades no lucrativas, Revista de Fomento Social, n. 260, p. 695-726, 2010.

MUÑOZ, R. M.; VALENCIA, P. T. Responsabilidad social y transparencia a través de la Web: un análisis aplicado a las cooperativas agroalimentarias españolas. Revista de Estudios Cooperativos, n. 114, p. 84-105, 2014.

NAVARRO, A,; ORTIZ , D.; ALCARAZ. Online dissemination of information on sustainability in regional governments.GovernmentInformationQuarterly, n. 33, v.1, p. 5366, 2016.

OLIVEIRA, F. F.; MOURA-LEITE, R. C. As práticas de responsabilidade social de empresas modelo em sustentabilidade. Revista Ciências Administrativas, v. 20, n. 1, p. 249284, 2014. 
ORGANIZAÇÃO DAS COOPERATIVAS BRASILEIRAS. Ramo de Atividades, números OCB 2017Recuperado em: http://www.ocb.org.br/\#/ramo-agropecuario. Acesso em: 30 maio. 2017.

ORGANIZAÇÃO DAS NAÇÕES UNIDAS PARA AGRICULTURA E ALIMENTAÇÃO (2011). Recuperado em: https://www.fao.org.br/cacrfp.asp Acesso em 20 jan. 2016.

PATIL, V. H.; SINGH, S. N.; MISHRA, S.; DONAVAN, D. T.Efficient theory development and factor retention criteria: Abandon the eigen value greater than one criterion. Journal of Business Research, V. 61, n.2, p. 162-170, 2008.

PAVIE, J. J. E. Responsabilidade social corporativa e desempenho financeiro: Uma metaanálise. 2008, 76 f. Dissertação (Mestrado Profissionalizante em Administração) - Curso de Pós-Graduação em Administração, Faculdades Ibmec, Rio de Janeiro, 2008.

RAHMAN, A. R.; DEBRECENY R. S. Institutionalized Online Access to Corporate Information and Cost of Equity Capital: A Cross-Country Analysis. Journal of Information Systems, v.28, n.1, p. 43-74, 2014.

SCHULTZ, F.; CASTELLÓ, I. E; MORSING, M. J. The construction of corporate social responsability in network societies: A comunication view. Journal of business ethics, v. 115 , p. 681-692, 2013.

SOLANO, S. E.; CASADO, P. P.; UREBA, S. F. Análisis de la divulgación de la información sobre la responsabilidad social corporativa en las empresas de transporte público urbano en España. Revista de Contabilidad, v. 19, n. 2,p. 195-203, 2016.

SPAREMBERGER, A.; ZAMBERLAN, L.; SAVOLDI, Z..A responsabilidade social em cooperativas agropecuárias do Rio Grande do Sul. Revista Capital Científico - Eletrônica, v. 8, n. 1, p. 107-120, 2010.

TOUS Z. D.; CIRUELA, L.A.M. Responsabilidad social y cultura en las sociedades cooperativas agrarias. Revista de Economía pública, social y cooperativa, n. 53, p.261-284, 2005 .

VIEIRA, K. M.; DALMORO, M. Dilemas na construção de escala de likert: o número de itens e a disposição influenciam nos resultados. Anais do Encontro Nacional da Associação Nacional de Pós-Graduação e Pesquisa em Administração, Rio de Janeiro, RJ, Brasil, 2008. 